\title{
A Decentralized and Proactive Architecture based on the Cyber Physical System Paradigm for Smart Transmission Grids Modelling, Monitoring and Control
}

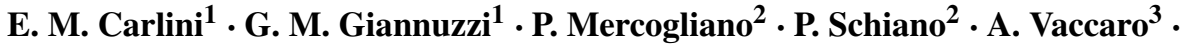 \\ D. Villacci ${ }^{3}$
}

Received: 12 June 2015 / Accepted: 2 February 2016 / Published online: 25 February 2016

(C) Springer Science+Business Media Singapore 2016

\begin{abstract}
The present paper presents a Cyber Physical Power System (CPPS) framework based on the ServiceOriented Architecture for proactive transmission grids control, modeling and monitoring. CPPS paradigm aims at integrating and coordinating computation, networking, and physical processes according to a holistic vision of the transmission system. The key feature of the proposed framework is the ability of multiple entities to process, manage and share massive heterogeneous information. More specifically, the idea is to conceptualize a holistic architecture that enables the computing resources to deliver much more automation that the sum of its individually self-managed components, allowing the Transmission System Operator to improve the interoperability and the integration level of monolithic and hard to customize power system control and monitoring functions. Moreover, it allows TSO to develop content-based data extraction and aggregation from a host of pervasive sensors network and to exploit distributed embedded computing resources aimed at solving large-scale problems. To assess the benefits of the CPPS framework, the first experimental results obtained on a real test bed are presented and discussed.
\end{abstract}

\section{A. Vaccaro}

vaccaro@unisannio.it

D. Villacci

villacci@unisannio.it

1 Italian Transmission System Operator, Terna Rete Italia SpA, Viale Egidio Galbani, 70 - 00156 Roma, Italy

2 Italian Aerospace Research Center, CIRA, Via Maiorise, 1, 81043 Capua, CE, Italy

3 Department of Engineering, University of Sannio, Piazza Roma 21, 82100, Benevento, Italy
Keywords Cyber physical system - Smart transmission grids . Proactive power systems · Decentralized smart grids computing

\section{Introduction}

European electrical transmission systems were traditionally characterized by the presence of different utilities, heterogeneous standards, overlapping geographical areas and a general lack of integration. Today, these highly interconnected systems are subject to a host of challenges.

In particular, the modern energy policy guidelines defined by the European Commission [1] are aimed at contrasting global warming through a massive deployment of Renewable and Distributed Energy Sources. This could sensibly raise the number of random power injections, which increase the complexities in transmission system operation, requiring larger spinning reserve margins, more effective voltage regulation strategies, and a more strict coordination with the Distribution System Operators (DSOs).

Further technical complexities have been induced on the transmission systems by the large-scale deployment of the deregulated energy markets, which are lowering the traditional boundaries among the national power systems, since the power transactions could take place anywhere in the global European power network under free market rules. This is introducing more uncertainties in short-term power systems operation, and making the transmission systems more vulnerable to dynamic perturbations.

Furthermore, the operation environment of future transmission networks is expected to become increasingly rigorous in Europe, due to the continually evolving functions of the national power systems from operation jurisdiction to control responsibly - coupled with the rising demand and expectation for power system reliability [2]. Finally, 
the upgrading of the transmission infrastructures, which is required to reliably supply the increasing European load demand and to support the massive pervasion of renewable power generators, should comply with new and more strictly environmental constraints, which impose the maximum exploitation of the existing assets before constructing new transmission facilities.

In this complex scenario, the intrinsic limitations characterizing the traditional power systems operation frameworks are clearly emerging, and Transmission Systems Operators (TSOs) are requested to enhance their governance systems in order to address the following issues:

- Improve the power system security analysis by evaluating the impact of multiple contingencies (i.e. N-2 criteria), and dynamic phenomena (i.e. dynamic security analysis);

- Conceptualize new monitoring paradigms aimed at supporting rapid decision in a data rich, but information limited environment, by processing the streams of data acquired by the field sensors, and providing TSO with the necessary information to act on in the timeframes necessary to minimize the impact of disturbances;

- Develop "plug and play" interoperability modules aimed at interfacing heterogeneous control and monitoring tools with the field sensors;

- Improve the exploitation of existing transmission assets by deploying more effective power components loading policies (i.e. dynamic loading), and enhanced probabilistic based analysis aimed at assessing the technical and economic risks of the load management policies, especially during emergency conditions;

- Design more effective tools for real-time information sharing between the TSOs, the asset managers of the power distribution systems and the market operators in order to allow all of them to plan coordinated corrective actions aimed at mitigating the effect of critical contingencies on the national power networks and reacting to predicted system outages [3].

To address these issues, the Cyber Physical Power System (CPPS) paradigm has been recognized as the most promising enabling technology for integrating and coordinating computing, networking, and physical processes according to a holistic vision of the transmission system [4]. In CPPS the network components and embedded computing entities interact and cooperate in order to $(i)$ pervasively collect synchronized information about the electrical variables (e.g. voltage and current phasors, active and reactive power) and meteorological parameters (e.g. network component and environmental temperature, wind speed and direction); (ii) process and amalgamate the previous information in order to identify proper control actions according to fixed objectives; (iii) dynamically adapt the parameters of the grid controllers via networked bio-inspired systems.

The cornerstone of CPPS is the ability of multiple entities (e.g. components, sensing units, software modules) to process, manage and share accurate and massive heterogeneous information. It follows that the development of a reliable, flexible and autonomic architecture for information processing represents a crucial issue in deploying the CPPS functions. This architecture aims at extracting domain specific features, complex relationships, and hypotheses from large heterogeneous networks of massive observations and simulated data generated by the CPPS applications and modules. This knowledge building process asks for converting data generated by heterogeneous sources (i.e. distributed measurement systems, energy management systems, numerical weather prediction models, producibility forecast models for RES powered plants etc.) into high-level information and eventually into actionable intelligence at different application domains (i.e. data analysis centres, research centres etc.). To address these needs, advanced "Information Services" aimed at providing the right information at the right moment to the right decision maker are required. This asks for conceptualizing a distributed fusion framework that allows the TSO to browse and access the heterogeneous data resources by providing functionalities for information management, storage, query, and inference services specifically designed for supporting the CPPS operation. This framework should be designed to enable resource discovery and to support the TSO to take rapid decisions in a data rich, but information limited environment. The latter issue represents a critical requirement in modern transmission system operation since it is expected that the large-scale deployment of the CPPS paradigm will massively increase the data exchange rate of several order of magnitude [5]. Consequently the streams of data acquired by the distributed grid sensors and/or generated by the domain specific mathematical models could not provide TSO with the necessary information in useful timeframes. Even in the presence of advanced tools aimed at converting the data into information, the TSO must face with the challenge of not having a full understanding of the information context and, therefore, the corresponding content cannot be used with any high degree of confidence. In deploying these information services, it will be incumbent upon the CPPS research community to conceptualize information paradigms aimed at facilitating operational data sharing in interoperable formats defined according to coordinated process chains.

In this context, the rapid development of the Information and Communication Technologies (ICT) has opened the door towards feasible and cost-effective control and monitoring paradigms, allowing more extensive intra/interutility information exchange, diffusion, and open access to a wide range of real time information [6]. Consequently, 
they could play a strategic role in supporting the deployment of advanced information services for CPPS as confirmed by various research studies recently published in the literature [7, 8]. Armed with such a vision, TERNA, the Italian TSO, has activated intensive experimental activities aimed at deploying advanced ICT based CPPS functionalities, which include synchronized measurement systems, adaptive protection schemes, expert based decision support systems, wide area monitoring and control systems $[9,10]$.

Although these functionalities demonstrated their effectiveness in improving the performances of the Italian transmission system, they are designed to address only a specific functionality, such as data acquisition or information processing, and do not deal with the concept of proactivity, which represents the most promising tool for dealing with the increasing uncertainties characterizing modern transmission networks. Moreover, the rigorous deployment of the CPPS paradigm asks for the definition of an integrated, scalable and pervasive framework aimed at executing very complex control and monitoring applications according to fixed time constraints, and built by composing the required CPPS functionalities in a standardized, easy-to-use and well-defined way.

To address these issues, in this paper an autonomic CPPS framework based on the Service-Oriented Architecture for proactive transmission grids control, modeling and monitoring is proposed. The idea is to conceptualize a holistic framework based on a collection of interactive web services, which enables the computing resources to deliver much more automation that the sum of its individually self-managed components. These information services describe a set of modular, and self-contained applications that could be remotely invoked by standard XML protocols, thus resulting independent from both the hardware/software platform and the programming language adopted for the service implementation $[11,12]$. This feature allows TSO to improve the interoperability and the integration level of monolithic and hard to customize power system control and monitoring functions, as far as the possibility to survive evolutionary changes in the structure and implementation of the internals of each CPPS function. Moreover, it allows developing content-based data extraction and aggregation from a host of pervasive sensors network, and exploiting distributed embedded computing resources aimed at solving large-scale problems.

Numerical results obtained on a real case study are presented and discussed in order to prove the effectiveness of the proposed CPPS framework in the task of solving complex and computational intensive power system operation problems.

\section{The CPPS Architecture}

The proposed CPPS framework is deployed according to the Service-Oriented Architecture, which is a paradigm that links-up heterogeneous information services by defining software interfaces that are independent from the hardware platform, the operating system, and the programming language adopted to implement the services. This feature allows the information services to cooperate and interact together, offering a set of intrinsic advantages such as high interoperability levels, integration of legacy power systems applications, adaptivity to power system evolution, information system survivability, and dynamic service reconfigurability. These features mainly derive by the deployment of advanced tools for information processing based on the Web Services technology, which extend the concept of software applications by introducing the notion of information service, representing a general computing

Fig. 1 The holistic CPPS architecture

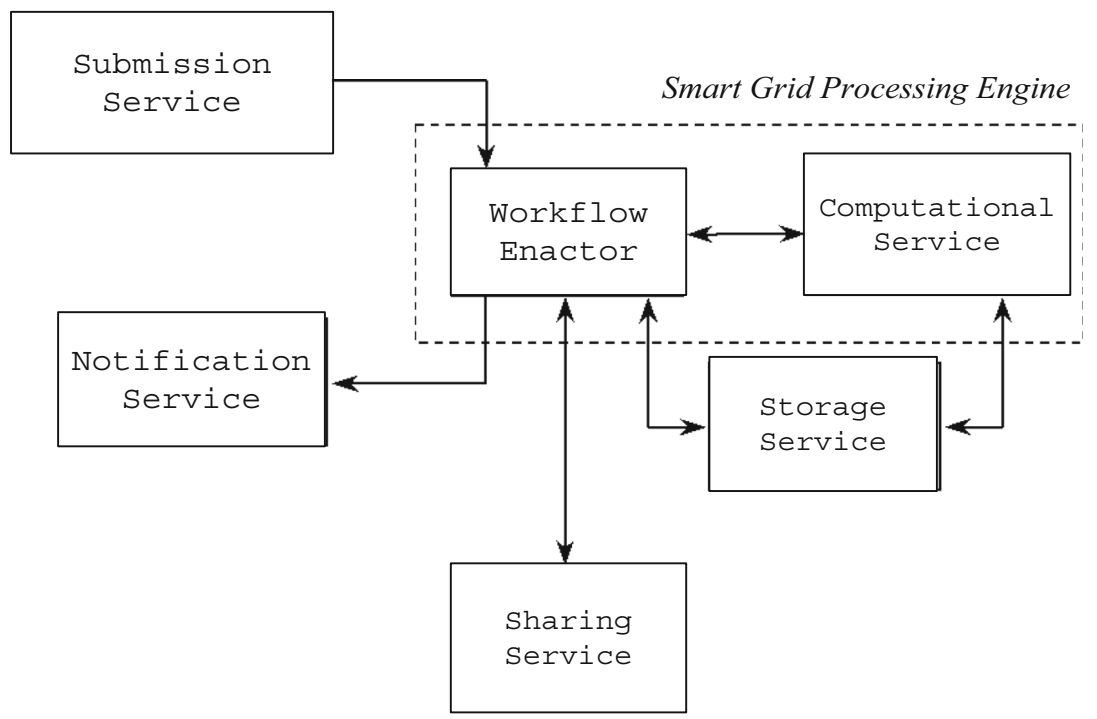


function that could be invoked by means of a software interface, defined independently from the programming language adopted to implement the function.

Hence, the main idea is to conceptualize a distributed meta-architecture for information processing in CPPS, which aims at abstracting, in a dynamic and geographically distributed computing scenario, the traditional centralized computing processing paradigm by means of interactive web services, as shown in Fig. 1.

The main component of this computing architecture is the Smart Grid Processing Engine, whose logical architecture is schematically depicted in Fig. 2, which orchestrates the following high-level information services:

- Submission Service, which handles both the user submission requests and the input data acquisitions;
- Computational Service, which performs the CPPS functions;

- Storage Service, which stores and manages the historical field data;

- Notification Service, which manages the output data;

- Sharing Service, which allows sharing information with other collaborative and cooperative frameworks.

In particular, the architecture of the Submission Service, which is schematically represented in Fig. 3, is based on the following Web Services:

1. FieldDataAcquisitionWs: it periodically acquires the field data useful for transmission system monitoring and control by querying the available set of information sources (i.e. SCADA systems,

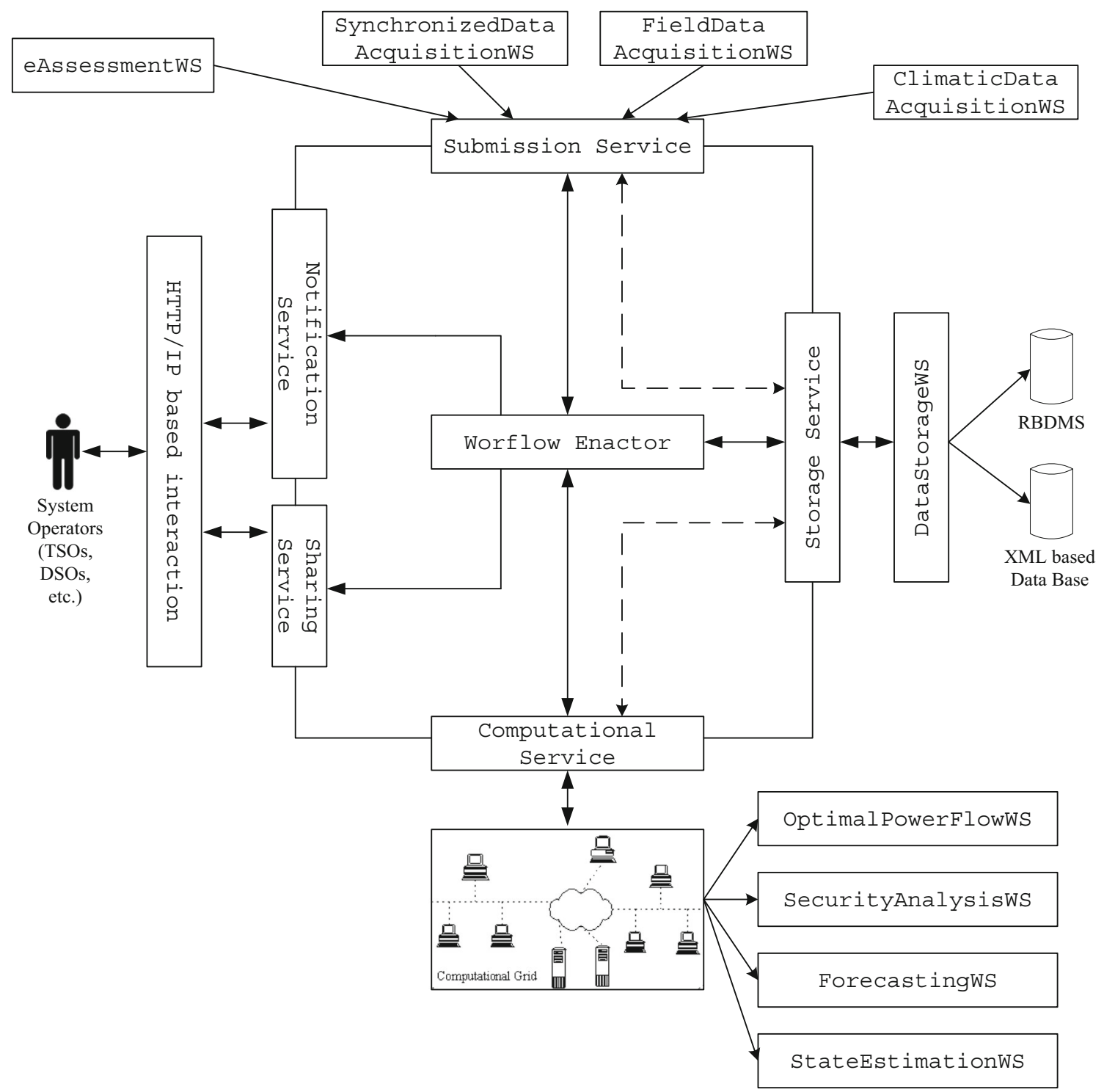

Fig. 2 The architecture of the Smart Grid Processing Engine 


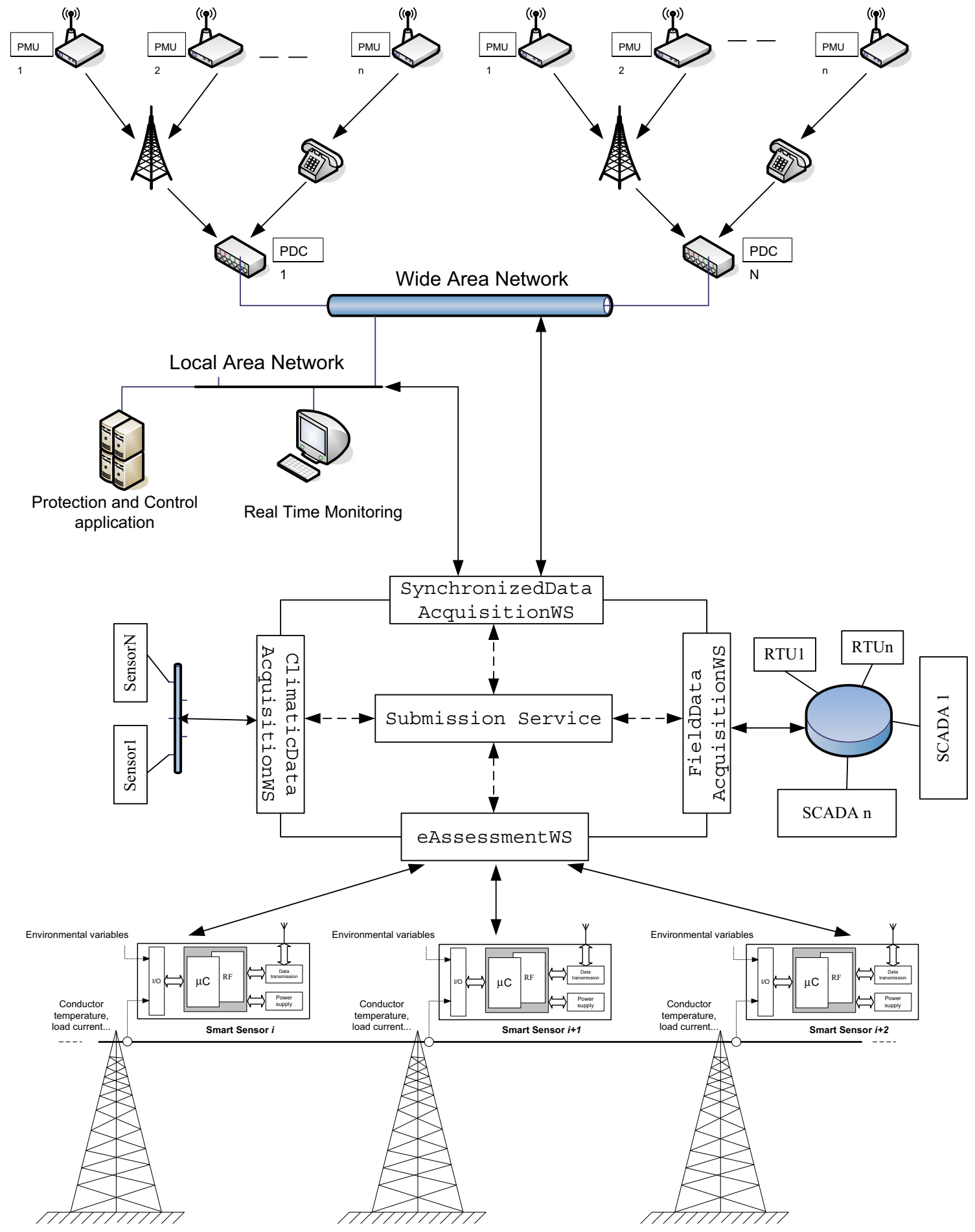

Fig. 3 The architecture of the Submission Service

Field Electrical Measurements, Remote Terminal Units);

2. MeteoDataAcquisitionWs: it acquires georeferential information on the forecast of the weather variables of interest for transmission system operation.
To this aim it interacts with weather measurement stations, meteorological Web Services and Geographical Information Systems (GIS);

3. SynchronizedDataAcquisitionws: it acquires synchronized information about voltage and current 
phasors, frequency and rate-of-change-of-frequency by interacting with synchronized Phase Measurement Units and Wide-Area Measurement Systems.

4. eAssessmentWS: it estimates the dynamic thermal ratings of the critical power components by interacting with a pervasive sensors network deployed on the transmission system components.

The Computational Service is deployed by a highly scalable computational grid [13] and it is based on the following interactive web services:

1. ForecastingWs:

a. Weather forecasting: it download, through an FTO service provided by the Italian Meteorological Service, forecast data from the European Center for Medium Range Weather Forecasting Integrated Forecast System and from the Italian local model COSMO. These forecasted data include the evolution of the main atmospheric variables (i.e. wind speed, environmental temperature) on selected geographic areas for time horizons raging from 3 to $72 \mathrm{~h}$.

b. RenewableEnergyForecasting: starting from the actual values of the meteorological variables acquired by the MeteoDataAcquisitionWS, it improves the accuracy of the corresponding forecasting profiles obtained by the Weather forecasting module by adopting an adaptive supervised learning system. The corresponding profiles are processed in order to forecast the power profiles generated by renewable power generators (i.e. wind generators) on time horizon ranging from 15 minutes (i.e. short time) to 1 day (i.e. medium time).

2. StateEstimationWS: it computes the voltage phasor at each electrical bus of the transmission network by properly processing the available set of measurements. The latter includes both data acquired by the FieldDataAcquisitionWS (e.g. real/reactive power flows, active and reactive power injections and bus voltages magnitudes) and synchronized measurements acquired by the SynchronizedDataAcquisitionWS.

3. SecurityAnalysisWs: starting from the current system state computed by the StateEstimationWS, the expected evolution of the load demand and the renewable power generation forecasted by the ForecastingWS and the actual thermal ratings of the main power components computed by the eAssessmentWS, it solves the power system state equations for the more probable operation scenarios in order to classify critical contingencies that could lead to system vulnerabilities.
4. OptimalPowerFlowWS: it solves optimal power flow problems aimed at supporting the TSO in identifying proper control actions aimed at managing the critical contingencies identified by the SecurityAnalysisWS.

The Sharing Service aims at allowing the TSO to interact and cooperate with external entities by sharing real time information. It asks for migrating the form based legacy systems characterising the existing control and monitoring frameworks towards service-oriented paradigms by adapting their legacy interfaces to the interaction paradigm defined by the following Web services:

1. DSOWS: it supports real time information exchanging between the TSO and the Distribution System Operators (DSO). They include vulnerability-related information, interdependencies, threat-related information, disaster-response and recovery actions.

2. GMEWS: it allows the TSO to interact with the Web Services designed by the Italian Power Exchange (IPEX).

The Storage Service is based on the DataStorageWS, which aims at permanently storing historical data and alarm conditions characterizing the transmissions system operation, and can be invoked by a data access middleware.

Finally, the Workflow Enactor aims at orchestrating the execution of the previously described interactive services, adding advanced proactive functionalities related to the specific CPPS application.

In the following sections, the main modules composing the CPPS architecture will be analyzed.

\section{Submission Service}

The Submission Service aims at acquiring and handling operational information in interoperable formats by supporting an effective knowledge (feature) extraction from heterogeneous and massive sensor data, which are acquired by pervasive grid sensors interfaced to the Supervisory Control And Data Acquisition systems (SCADA) and to the Energy Management Systems.

\section{FieldDataAcquisitionWS}

It is responsible for the on-line acquisition of the field data by interacting with the available SCADA systems, remote terminal units and intelligent electronic devices by standardized XML messaging. This service delivers the getBusMeasurements portType, that receives, as input, the set of buses and, for each of them, the kind of available measurements, and returns, as output, the required values. All the possible kinds of measurements are welldefined, in particular for each kind of parameter a code and 
a basic measurement unit are defined. Each measurement value returned by the FieldDataAcquisitionWs is associated to a timestamp, which indicates the UTC time at which the corresponding variables were measured. The returned type by the described portType is a String, that corresponds to the URL of the file containing the required information.

\section{MeteoDataAcquisitionWS}

This service aims at acquiring the evolution of the main meteorological variables on the transmission system by interacting with a network of pervasive and distributed meteorological stations. The latter are equipped with wind speed and direction sensors, environmental temperature sensors, solar irradiance sensors, humidity and pressure sensors, which are geographically distributed in the most critical sections of the Italian transmission systems.

\section{SynchronizedDataAcquisitionWS}

This service, whose architecture is schematically depicted in Fig. 4a, aims at acquiring and processing the available set of synchrophasor by interfacing the CPPS with the Phasor Measurement Units (PMUs), the Phasor Data Concentrators (PDCs) and the computing facilities of the Italian Wide Area Monitoring (WAM) system. This sophisticated monitoring infrastructure, whose architecture is depicted in Fig. 4b, was designed to improve the early detection of critical power system conditions, by a more accurate and reliable state estimation, to reduce the probability of catastrophic events, by enhancing the monitoring of the dynamical interactions with the other national power systems, and to mitigate the impacts of critical contingencies, by minimizing the reaction times to both exogenous and endogenous disturbances [14].

To deploy these advances functionalities the monitoring system integrates up to 60 PMU devices, connected by a private communication network based on Direct Numerical Circuit (DNC) channels, and is equipped with software interfaces based on the IEEE1344 and C-37.118 protocols, which allow to exchange real-time data with the WAMS of three European TSO. Thanks to these features, the system is able to acquire, process and store detailed synchrophasor data characterizing the whole ENTSO-e CESA dynamic behavior, which allow the National Control Center (NCC) to detect real-time trends and alarms related to [14]:

- $\quad$ voltage magnitude (low/high) ;

- frequency (low/high) ;

- angle difference (high);

- damping (low; from online oscillatory analysis function);
- voltage collapse;

- under-frequency load shedding (from load shedding evaluation function);

- islanding, loss of synchronism, and frequency stability;

An interesting features characterizing the Italian WAMS is its ability to share the synchronized data with external processes via proprietary or standard protocols (i.e. Object Linking and Embedding for Process Control-OPC) as far as real-time applications, distributed processing systems, external computing systems (i.e. equipped with MATLAB ${ }^{\circledR}$ ) and visualization software are concerned.

These information-sharing functions have been exploited to interface the WAMS to the CPPS in order to allow the WAM system to evolve toward a Wide Area Monitoring, Protection and Control system (WAMPAC). The latter has been recognized as one of the more promising enabling technology to deploy advanced CPPS functions such as [15]:

- System Integrity Protection Schemes;

- Distribution circuits network management;

- Dynamical loading of power equipment;

- System restoration and smart restoration tools;

- Advance warning systems of impending trouble.

\section{eAssessmentWS}

It interacts with a network of cooperative smart sensors deployed on the critical power components to predict the true load capability curves, which report the magnitude and the time duration of the electrical loads that the components (i.e. overhead lines and power transformers) can effectively and reliably support [16]. To this aim, the smart sensors:

- acquire the component temperature by querying a set of local temperature sensors;

- check for the consistency of the measured temperatures;

- estimate the component thermal parameters and the weather conditions;

- compute the real load capability curves;

- spread the corresponding results along the sensors network by a decentralized gossiping algorithm.

Thanks to these features the eAssessmentWS can obtain the load capability curves of the monitored component by enquiring any smart sensor.

\section{Computational Service}

To support the deployment of the CPPS applications, online computations are required. These include spatial and temporal forecasting analysis, multi-sensor data fusion, spatial data correlation, data mining, statistics analysis and static/dynamic network modeling. 


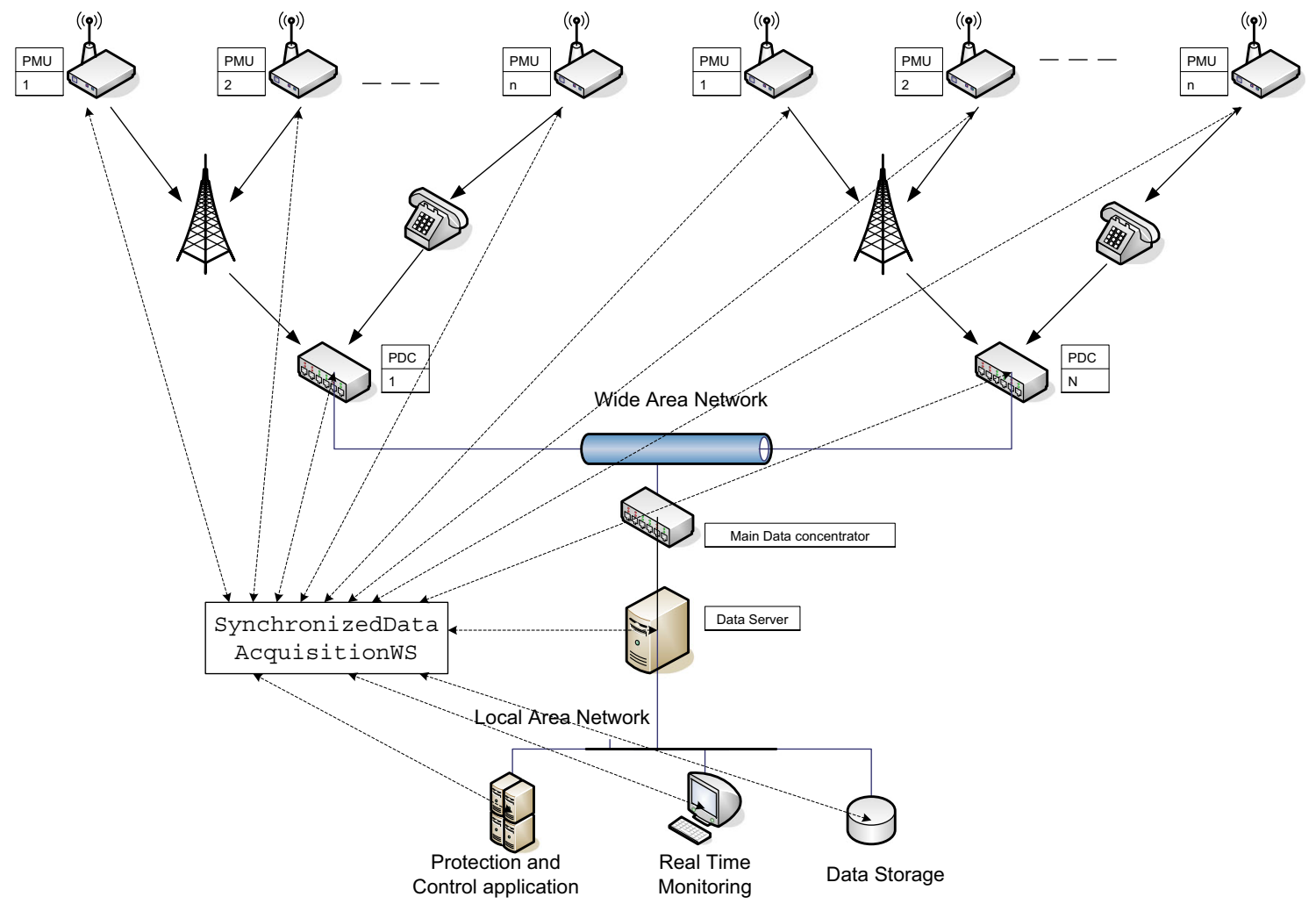

a)

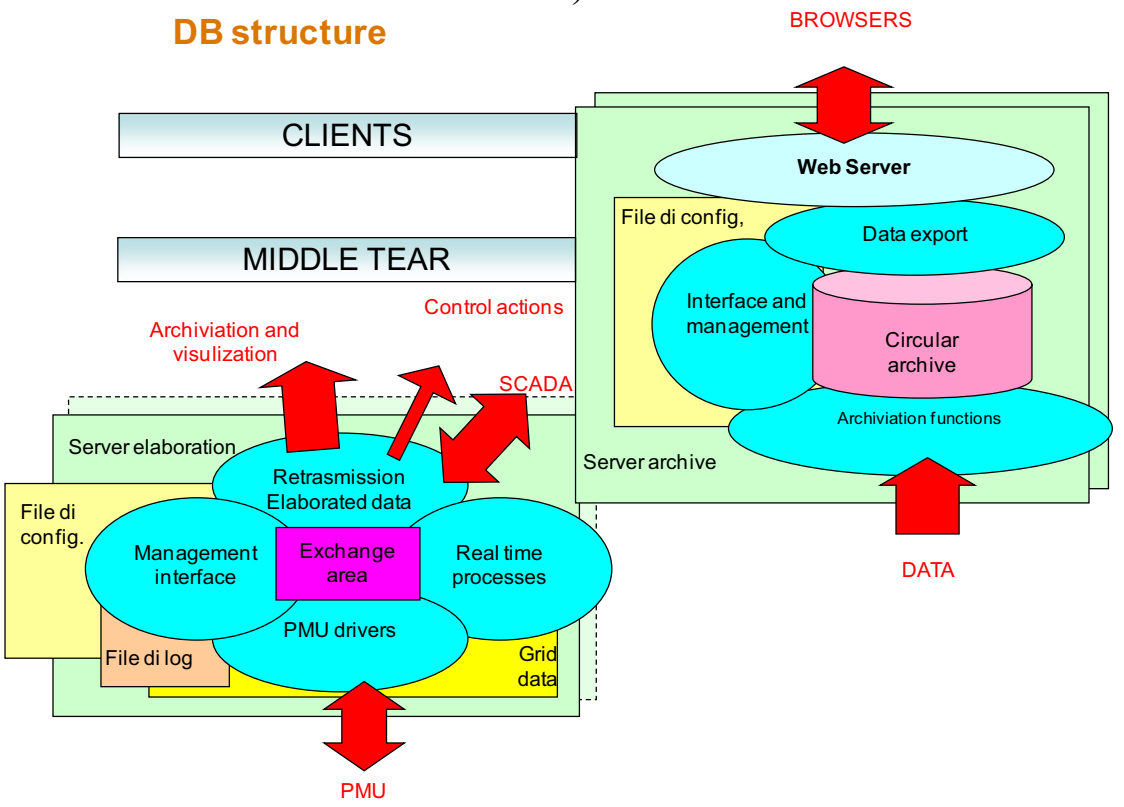

b)

Fig. 4 Interaction of the CPPS with WAMS: a Architecture of the SynchronizedDataAcquisitionWS b Architecture of the Italian WAMS [14]

To address these computationally demanding analyses in useful execution times, the Computational Service employs parallel algorithms deployed on a highly scalable, distributed and dynamically extensible computing engine [17].
The adoption of this grid computing based paradigm allows TSO to solve complex control and monitoring functions in fixed time constraints by dynamically exploiting distributed computing resources, and overcoming the intrinsic 
limitations of static parallel networking environments, which could not assure enough scalability in executing intensive CPPS calculations.

The interface between the Computational Service and the computational engine is realized by invoking a specific Web Service, namely the JobSubmissionWS, which exports the set of brokering functionalities aimed at submitting the computing tasks and managing the corresponding results [18]. Thanks to the adoption of this service, the following CPPS applications have been developed.

\section{ForecastingWS}

This Web Service invokes the WeatherForecasting function to acquire the forecasted profiles of the main meteorological variables computed by the European Center for Medium Range Weather Forecasting Integrated Forecast System for time horizons ranging from 3 to $72 \mathrm{~h}$. These profiles are obtained by solving the primitive equations of the atmospheric General Circulation Model, which takes into account the main physical interactions like ocean and atmosphere, soil wetness and atmosphere and snow covering and atmosphere [19]. This model contains 91 layers between surface and $0.01 \mathrm{hPa}$ (about $80 \mathrm{~km}$ ) pressure level in the vertical space, the resolution of model is about $16 \mathrm{~km}$ in horizontal space, and the temporal resolution is 12 minutes. More details on the ECMWF model can be found in [20].

The forecasted profiles acquired by the WeatherForecasting function and the meteorological data acquired by the
MeteoDataAcquisitionWS are amalgamated by the RenewableEnergyForecasting function in order to forecast the power profiles generated by the wind farms connected to the transmission network. This amalgamation process allows characterizing the performances of the wind farms in function of the orographic conformations of the installation sites. This is obtained by deploying the "Meteo-Energy Chain" paradigm depicted in Fig. 5, which allows the TSO to accurately forecast the wind power profiles on a medium/long term scenario by integrating satellite data processing, advanced modeling techniques and machine learning tools.

The adopted forecasting approach pursues the effective integration of the physical knowledge available from the WeatherForecasting function with the additional information retrievable from experimental measurements acquired by the MeteoDataAcquisitionWS. The basic idea is to reduce the systematic error over the geographical point of interest characterizing the numerical weather prediction model, by deploying an "optimization" framework, which periodically compares the forecast data and the observation, and improves the prediction identifying proper correction factors for the different atmospheric variables by applying different statistical techniques. By comparing the performances of these corrected forecasted models over long time series, the framework select the specific method that, for the selected point and variable, permit to have the lowest forecasting. In the current version of this framework, twelve different methods have been considered.
Fig. 5 Meteo-Energetic Chain in ForecastingWS

\section{Meteo -Energetic Chain}

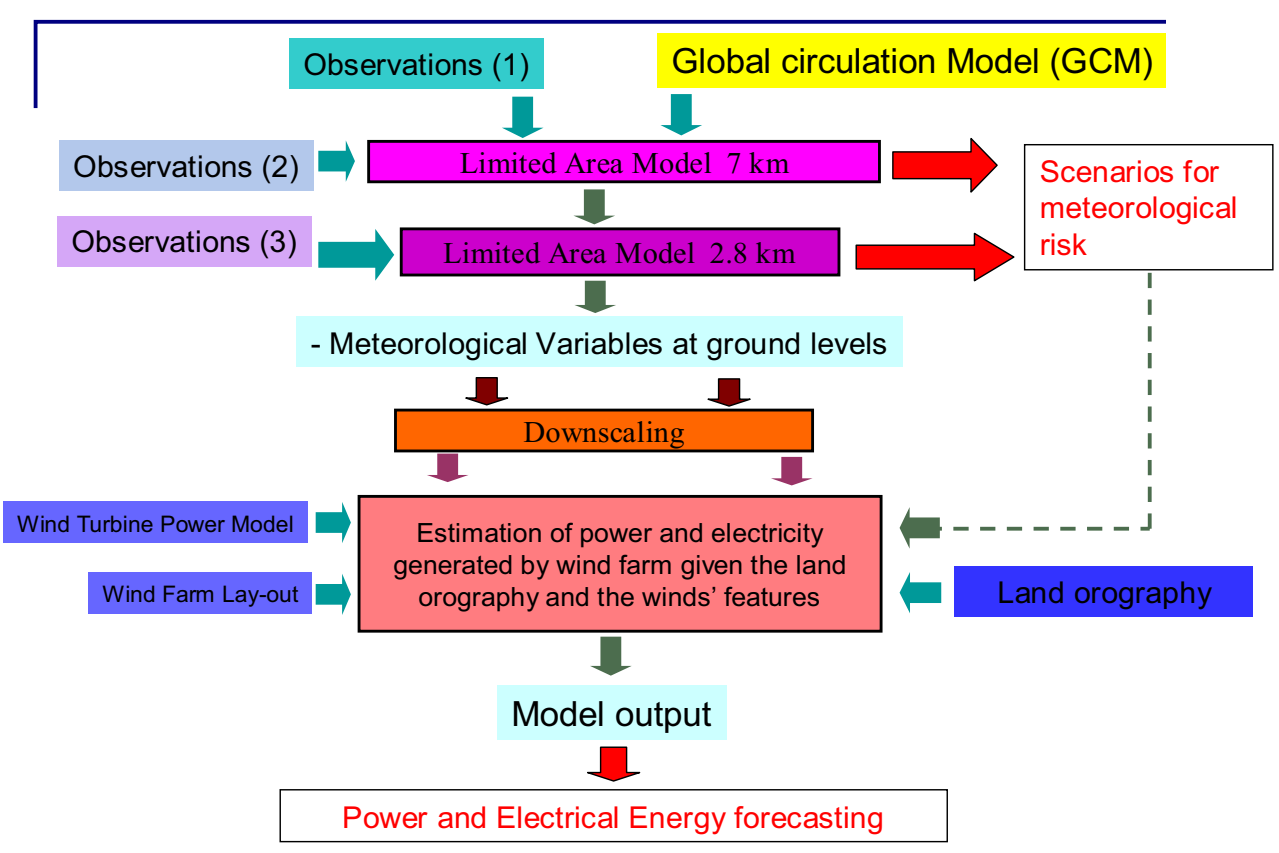


This forecasting architecture is expected to be reliable both in terms of robustness and speed, since it combines a reliable physical model with an adaptive dynamic corrector. The corrector model is sequentially adjourned, in order to adapt the whole architecture to "new" operating conditions. A way to detect a new condition is checking regularly the forecasting accuracy on fresh measurements: a forecasting error over a fixed threshold could be used to trigger the dataset update. It is worth noting also that this structure, by avoiding recursive feedbacks, should prevent stability problems and the consequent divergence of the forecasting errors.

\section{StateEstimationWS}

It computes the voltage phasor at each electrical bus of the transmission network by properly processing the available set of measurements acquired by the FieldDataAcquisitionWS and the SynchronizedDataAcquisitionWS. To solve this problem, this service deploy the solution paradigm proposed in [21], which aims at formulating the state estimation problem by a set of ordinary differential equations, whose equilibrium points represent the problem solutions. According to this solution paradigm, the power system state can be estimated by computing the quiescent state of an artificial dynamic model, which can be designed to be stable with an asymptotical convergence to an equilibrium point, and quite insensitive to many factors that can cause numerical instabilities to the traditional solution algorithms. These important features allow the TSO to overcome some of the inherent limitations of iterative minimization algorithms that can fail to converge due to the highly nonlinearities of the first-order conditions. Moreover, the overall state estimation framework is characterized by an intrinsic filter capability that could be very useful in solving the state estimation problem in the presence of uncertain, noisy or redundant data [22].

\section{SecurityAnalysisWS}

The on-line assessment of the static and dynamic security analysis is performed according to the main functional steps, and the logical information flows described by the UML activity diagram proposed in [13]. Initially, the SecurityAnalysisWS acquire the current power system state computed by the StateEstimationWS, the renewable power generation forecasted by the ForecastingWS, and the actual thermal ratings of the main power components computed by the eAssessmentWS. Then, starting from these data, it identifies the list of the most credible contingencies, generating the corresponding input files describing the input scenarios. This file is then used to solve the power system equations, and the corresponding results are analyzed in order to check if the network technical limits, i.e. buses voltage magnitudes, power lines limits and power equipment loading, are violated.

\section{OptimalPowerFlowWS}

The knowledge discovery on massive and heterogeneous field data developed by the ForecastingWS and eAssessmentWS allows us to define the OptimalPowerFlowWS which aims at reformulating the traditional Optimal Power Flow (OPF) problems according to a proactive and hybrid paradigm. The rationale inside this Web Service is to solve the OPF by considering the actual and the predicted profiles of the system variables (e.g. active power generated by renewable power generators, load demand, the power components hot spot temperature) and by properly modelling the effect of the exogenous variables on the power system behaviour (e.g. the climatic variables, the spot energy prices).

The latter concept could be deployed by directly integrating the heat balance equations (HBEs) [23, 24] describing the thermal dynamic of the power components in order to exploit their thermal inertia. The resulting coupled electrothermal framework, here referred as electro-thermal-OPF (ET-OPF), is based upon the so called electro-thermal coordination (ETC) concept which basically coordinates the transmission lines electrical and thermal behaviour, by employing a proper line electro-thermal model, in order to search for higher system exploitation levels.

The main difference of the ET-OPF model, with respect to a standard OPF model, relies on the inclusion of the HBEs, equal in number to the specific power components (i.e. overhead lines) subject to thermal monitoring, expressed as further problem equality constraints thanks to a proper numerical discretization (e.g. through an Euler method). Therefore, the conductor temperature of the monitored power lines becomes a specific problem decision variable at each integration step. Equation 1 reports the HBE general formulation which describes the dynamic behaviour of the line temperature $T_{l}(t)$ along the time. More specifically, the left hand side represents the thermal power stored per unit of length by the line, evaluated as product of the temperature derivative with respect to the time and the line heat capacity $\rho_{l}$. The term $\Gamma_{l}\left(T_{l}(t)\right)$ represents the single conductor real power losses per unit of length, $q_{l}^{s}(t), q_{l}^{c}\left(T_{l}(t)\right), q_{l}^{r}\left(T_{l}(t)\right)$ instead respectively represent the solar heat gain, the convection heat loss and the radiated heat loss.

$\rho_{1} \frac{\mathrm{d}}{\mathrm{dt}} \mathrm{T}_{1}(\mathrm{t})=\Gamma_{1}\left(\mathrm{~T}_{1}(\mathrm{t})\right)+\mathrm{q}_{1}^{\mathrm{s}}(\mathrm{t})-\mathrm{q}_{1}^{\mathrm{c}}\left(\mathrm{T}_{1}(\mathrm{t})\right)-\mathrm{q}_{1}^{\mathrm{r}}\left(\mathrm{T}_{1}(\mathrm{t})\right)$ 
The following approximated relationships can be employed for calculating the convective and radiative heat flux:

$\mathrm{q}_{1}^{\mathrm{c}}\left(\mathrm{T}_{1}(\mathrm{t})\right)=\mathrm{A}_{1}^{\mathrm{c}}\left(\omega_{1}(\mathrm{t})\right)\left(\mathrm{T}_{1}(\mathrm{t})-\mathrm{T}_{1}^{\alpha}(\mathrm{t})\right)$

$\mathrm{q}_{1}^{\mathrm{r}}\left(\mathrm{T}_{1}(\mathrm{t})\right)=\mathrm{A}_{1}^{\mathrm{r}}\left(\omega_{1}(\mathrm{t})\right)\left(\mathrm{T}_{1}^{4}(\mathrm{t})-\left[\mathrm{T}_{1}^{\alpha}(\mathrm{t})\right]^{4}\right)$

Except for the line reactance, whose functional dependence on the conductor temperature $T_{l}(t)$ is negligible [24], the remaining lumped model line parameters depend on it, through the relationships (4) (5) and (6):

$\mathrm{R}_{1}\left(\mathrm{~T}_{1}(\mathrm{t})\right)=\mathrm{R}_{1}^{\mathrm{ref}}\left[1+\alpha_{1}^{\mathrm{ref}}\left(\mathrm{T}_{1}(\mathrm{t})-\mathrm{T}_{1}^{\mathrm{ref}}\right)\right]$

$\mathrm{G}_{1}\left(\mathrm{~T}_{1}(\mathrm{t})\right)=\frac{\mathrm{R}_{1}\left(\mathrm{~T}_{1}(\mathrm{t})\right)}{\mathrm{R}_{1}^{2}\left(\mathrm{~T}_{1}(\mathrm{t})\right)+\mathrm{X}_{1}^{2}}$

$\mathrm{B}_{1}\left(\mathrm{~T}_{1}(\mathrm{t})\right)=\frac{-\mathrm{X}_{1}}{\mathrm{R}_{1}^{2}\left(\mathrm{~T}_{1}(\mathrm{t})\right)+\mathrm{X}_{1}^{2}}$

The three-phase active power transfer along a generic transmission line, interconnected between two generic network nodes, $m$ e $n$, can be determined by employing the following equation:

$$
\begin{aligned}
\mathrm{P}_{\mathrm{f} 1}(\mathrm{~V}(\mathrm{t}), \delta(\mathrm{t}), \mathrm{T}(\mathrm{t}))= & \mathrm{G}_{1}\left(\mathrm{~T}_{1}(\mathrm{t})\right) \mathrm{V}_{\mathrm{m}}^{2}(\mathrm{t})-\mathrm{V}_{\mathrm{m}}(\mathrm{t}) \mathrm{V}_{\mathrm{n}}(\mathrm{t}) \\
& \times\left[\mathrm{G}_{1}\left(\mathrm{~T}_{1}(\mathrm{t}) \cos \delta_{1}(\mathrm{t})\right)\right. \\
& \left.\quad-\mathrm{B}_{1}\left(\mathrm{~T}_{1}(\mathrm{t}) \sin \delta_{1}(\mathrm{t})\right)\right]
\end{aligned}
$$

The related three phase power losses due to Joule effect can be calculated through the Eq. 8; this value simply scaled from a factor $3 D_{l}$, with $D_{l}$ the conductor length, furnishes the quote $\Gamma_{1}=\mathrm{L}_{1} / 3 \mathrm{D}_{1}$ to use in Eq. 1 :

$$
\begin{aligned}
\mathrm{L}_{\mathrm{l}}(\mathrm{V}(\mathrm{t}), \delta(\mathrm{t}), \mathrm{T}(\mathrm{t}))=\mathrm{G}_{\mathrm{l}}(\mathrm{T}(\mathrm{t}))[ & \mathrm{V}_{\mathrm{n}}^{2}(\mathrm{t})+\mathrm{V}_{\mathrm{m}}^{2}(\mathrm{t}) \\
& \left.-2 \mathrm{~V}_{\mathrm{n}}(\mathrm{t}) \mathrm{V}_{\mathrm{m}}(\mathrm{t}) \cos \delta_{1}(\mathrm{t})\right]
\end{aligned}
$$

Below, the mathematical framework of the ET-OPF problem in the context of the day-ahead energy market-clearing problem, is formalized:

$$
\underset{\mathrm{P}_{\mathrm{g}}, \delta, \mathrm{T}}{\operatorname{Minimize}} \sum_{\mathrm{j}=1}^{\mathrm{N}_{\mathrm{CS}}} \sum_{\mathrm{i}=1}^{\mathrm{N}_{\mathrm{B}}} \mathrm{C}_{\mathrm{i}} \mathrm{P}_{\mathrm{gi}}(\mathrm{t})
$$

subject to

$$
\begin{aligned}
& \mathrm{P}_{\mathrm{g}}(\mathrm{t})-\mathrm{P}_{\mathrm{d}}(\mathrm{t})=[\mathrm{K}]^{\mathrm{T}} \mathrm{P}_{\mathrm{f}}(\mathrm{t}) \\
& {[\chi](\mathrm{T}(\mathrm{t})-\mathrm{T}(\mathrm{t}-\Delta \mathrm{t}))=\Gamma(\mathrm{t})+\mathrm{q}(\mathrm{t})+\Gamma(\mathrm{t}-\Delta \mathrm{t})+\mathrm{q}(\mathrm{t}-\Delta \mathrm{t})} \\
& -\mathrm{P}_{\mathrm{fN}}^{\max } \leq \mathrm{P}_{\mathrm{fN}}(\mathrm{t}) \leq \mathrm{P}_{\mathrm{fN}}^{\max } \\
& \mathrm{T}(\mathrm{t}) \leq \mathrm{T}^{\max } \\
& \mathrm{P}_{\mathrm{g}}^{\min } \leq \mathrm{P}_{\mathrm{g}}(\mathrm{t}) \leq \mathrm{P}_{\mathrm{g}}^{\max }
\end{aligned}
$$

$-\tau_{\mathrm{r}} \mathrm{r}_{\mathrm{g}}^{\max } \leq \mathrm{P}_{\mathrm{g}}(\mathrm{t})-\mathrm{P}_{\mathrm{g}}(\mathrm{t}-\Delta \mathrm{t}) \leq \tau_{\mathrm{r}} \mathrm{r}_{\mathrm{g}}^{\max }$

with initial conditions

$\mathrm{P}_{\mathrm{g}}(0)=\mathrm{P}_{\mathrm{g}}^{\text {in }} \delta(0)=\delta^{\text {in }} \mathrm{T}(0)=\mathrm{T}^{\text {in }}$

The meaning of the parameters, functions and variables involved in the presented framework is discussed in the following:

- $C_{i}$ is the cost of generatori;

- $\mathrm{P}_{\mathrm{g}}(\mathrm{t})$ represents the vector of generator outputs at time $t$;

- $\mathrm{P}_{\mathrm{d}}(\mathrm{t})$ represents the vector of bus demand at time $t$;

- $\mathrm{P}_{\mathrm{f}}(\mathrm{t})$ is the vector of sending and receiving line flows at time $t$;

- $[K]$ is the matrix mapping line flows to buses;

- Tis the vector of line temperatures at time $t$;

- $[\chi]$ is the diagonal matrix, whose nonzero elements are given by $\chi_{1}=2 \rho_{1} / \Delta$, whereas the net heat rate vector $\mathrm{q}(\mathrm{t})$ is defined by $\mathrm{q}(\mathrm{t})=\mathrm{q}^{\mathrm{s}}(\mathrm{t})-\mathrm{q}^{\mathrm{c}}(\mathrm{T}(\mathrm{t}))-\mathrm{q}^{\mathrm{r}}(\mathrm{T}(\mathrm{t}))$;

- $\mathrm{P}_{\mathrm{fN}}^{\max }(t)$ is the vector of maximum allowed line power flows for lines that are not thermally limited;

- $\quad P_{g}^{\max }(t)$ represents the vector of maximum allowed generation output at time $t$

- $\quad \mathrm{P}_{\mathrm{g}}^{\min }(\mathrm{t})$ represents the vector of minimum allowed generation output at time $t$

- $\mathrm{r}_{\mathrm{g}}^{\mathrm{max}}$ is the vector of maximum allowed generator ramp rates;

- $\tau_{\mathrm{c}}$ is the coordination period length;

The expected advantages resulting from the deployment of this hybrid OPF paradigm are:

- The line electro-thermal model employment for strategic interconnections like those among market zones could offer significant economic benefits for the entire community due to reduction of market splitting and hence of the electricity price paid by the customers;

- The ET-OPF solution, calculated by considering the additional extra capacity offered by the lines subject to dynamic thermal rating, could generally lead to dispatch less-expensive generators, with further economic benefits with respect to conventional OPF model;

- Re-definition of the maintenance programs taking into consideration the maximum line disconnection time, evaluated as the one at which the maximum allowable temperature is reached for a certain loading condition;

- Intelligent and rational use of the line transfer capability, seen as an actual resource to exploit along a time horizon on which the load profile is established or anyway deducted through forecast. 
Fig. 6 The congested power system analyzed in the experimental studies

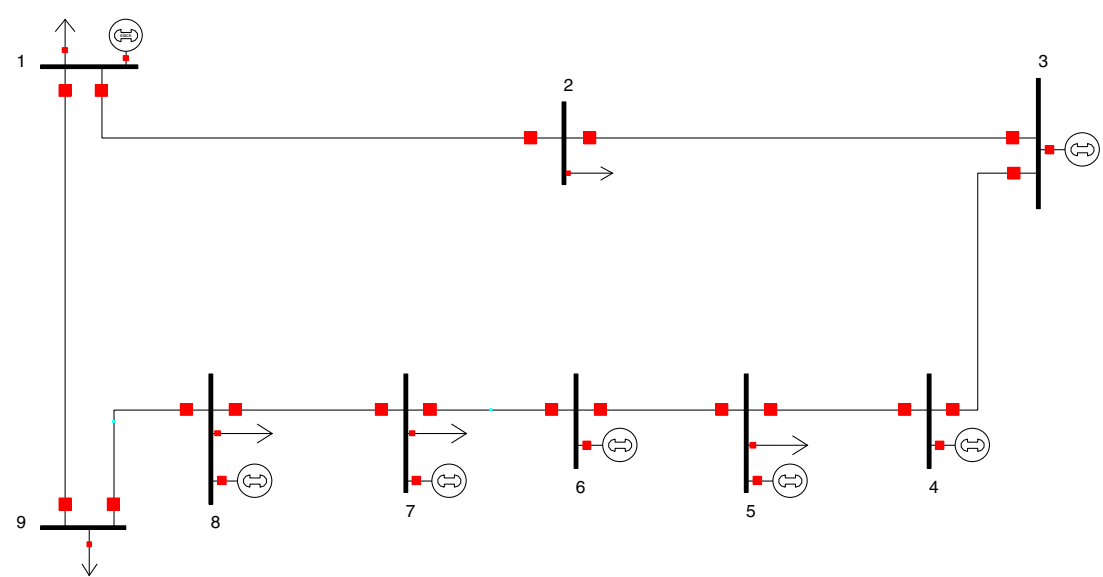

\section{Storage Service}

Storage systems are extremely useful in transmission system analysis, since they make available historical information that can be adopted in network planning or in designing a knowledge base for decision support systems. Hence, a specific Web Service, namely the DataStorageWS, has been designed to access and manage distributed and heterogeneous data storage resources, including Relational Data Base Management Systems (RDBMS) and XML database repositories.

To this aim, the OGSA-DAI has been considered in this research, since it allows to access and analyze distributed and massive data resources by a well-defined Grid Services interface, which is an instance of the Grid Data Service Specification defined by the Database Access and Integration Working Group of the Global Grid Forum.

\section{Information Sharing}

This service is implemented by two interactive web services, namely the DSOWS, which aims at supporting real time information exchanging between the TSO and the DSOs, and the GMEWS, which aims at allowing the TSO to interact with the Italian Power Exchange.

The information shared by these web services include:

- Vulnerability-related information, i.e. contingencies that could be critical for the security and reliability of the interconnected power systems;

- Interdependencies, i.e. information describing the effects of impending troubles;

- Threat-related information, i.e. information describing the effect of wide-area disturbances;

- Disaster-response and recovery plans.

\section{Hardware Deployment}

To assess the effectiveness of the CPPS framework numerical results obtained on a real case study are presented and discussed in this section. The considered power network, which is schematically depicted in Fig. 6, represents a $150 \mathrm{kV}$ equivalent portion of the Italian power system, which is frequently congested due to the large quantity of electrical power generated by the wind power plants installed in the area.

The overhead line connecting the buses 2 and 3 , which is frequently thermal congested and reduces the capability of the system to exploit the large renewable power generation available at bus 3 , represents the most critical section of this power system.

To improve the exploitation of the renewable power generation, without affecting the reliable power system operation, the proposed CPPS framework has been deployed. To this aim, the critical overhead line has been equipped by a thermal monitoring system, which integrates 2 temperature sensors installed in the most critical line sections and whose main characteristics are summarized in Table 1, and two Phasor Measurements Units installed to both ends of the line.

Table 1 Characteristics of the power Donut ${ }^{\circledR}$

\begin{tabular}{ll}
\hline Minimum start up current & $50 \mathrm{~A}$ \\
Current range & $0-3000 \mathrm{~A}+/-0.5 \%$ of reading \\
Conductor temperature & $-50{ }^{\circ} \mathrm{C}$ to $150{ }^{\circ} \mathrm{C}+/-0.5 \%$ of reading \\
Inclination & -15 deg tilt to +15 deg tilt $+/-0.025 \mathrm{deg}$ \\
Voltage & $1 \mathrm{kV}$ to $500 \mathrm{kV}-$ needs field scaling \\
Diameter & $320 \mathrm{~mm}$ \\
Width & $140 \mathrm{~mm}$ \\
Weight & $9,2 \mathrm{~kg}$
\end{tabular}




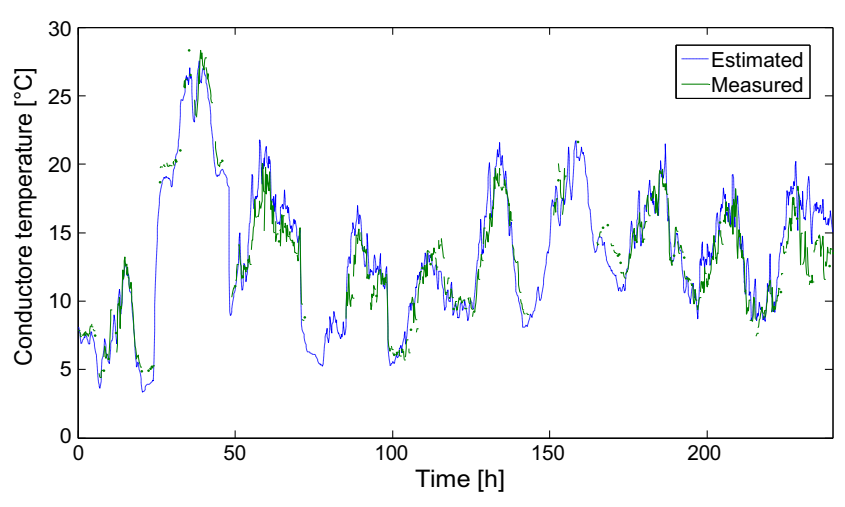

Fig. 7 Measured and predicted hot-spot temperature on the congested overhead line

The data measured by these sensors were acquired and processed by the eAssessmentWS and the SynchronizedDataAcquisitionWS, in order to estimate the hot-spot temperature of the overhead line and to calibrate the conductor thermal model parameters. The obtained results for a 10 days time scenario are summarized in Fig. 7, where the measured hot-spot temperature and the corresponding estimation computed by the calibrated thermal model are depicted.

The analysis of these results confirms the good accuracy of the eAssessmentWS, which aims at continuously adapting the conductor thermal model parameters in order to properly describe the intrinsic time-varying nature of the complex thermal phenomena. Starting from these data the maximum allowable duration for each hypothetical loading condition can be computed by processing the measured hot-spot conductor temperature, the weather variables forecasted along the line route by the ForecastingWS and the maximum allowable conductor temperature. The results of this computing process for a particular conductor thermal state, which is characterized by a hot-spot temperature of

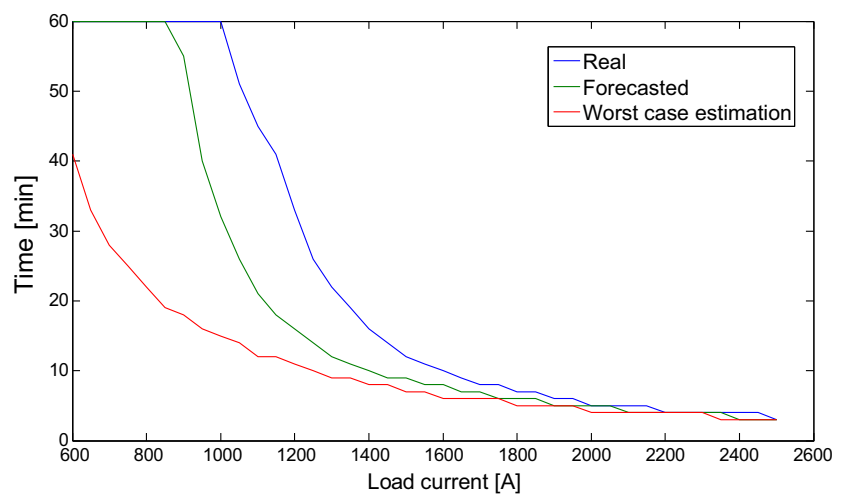

Fig. 8 Load capability curve estimated by the CPPS framework $20^{\circ} \mathrm{C}$, and by considering a maximum allowable conductor temperature of $70{ }^{\circ} \mathrm{C}$, is depicted in Fig. 8. In this figure the load capability curve predicted by the CPPS framework has been compared with the real curve, which has been computed by processing the measured environmental variables, and the worst case estimation adopted by the TSO in defining the line loading strategies, which has been computed by assuming zero wind speed and an environmental temperature of $30^{\circ} \mathrm{C}$. The comparison between these profiles confirm the great benefits deriving by the deployment of the CPPS framework, which allow to reliable improve the line exploitation.

Starting from the computed load capability curve, and the forecasted wind power profiles computed at each bus by the ForecastingWS, the ET-OPF problem has been solved, and the obtained solution has been compared to those obtained by solving the same problem, but considering the maximum load current constrained to $980 \mathrm{~A}$ for all the lines, here referred as worst-case operation.

In particular, a 6-hour operation scenario has been considered in this analysis, and the overall problem was to identify the hourly dispatch of the wind generators, which minimizes the integral of the difference between the available, and the dispatched wind power profiles, and satisfies both the power flow equations, and the component operation constraints. The latter are describes by a thermal constraint of $60^{\circ} \mathrm{C}$ on the monitored line, and a current constraint of $980 \mathrm{~A}$ on the other lines, which is the static thermal rating assumed by the TSO during winter operation.

The obtained results have been summarized in Figs. 9 and 10, with Fig. 9 depicting the hourly power dispatch profiles of the largest wind generator, and Fig. 10 reporting the hourly load current profiles for the congested line.

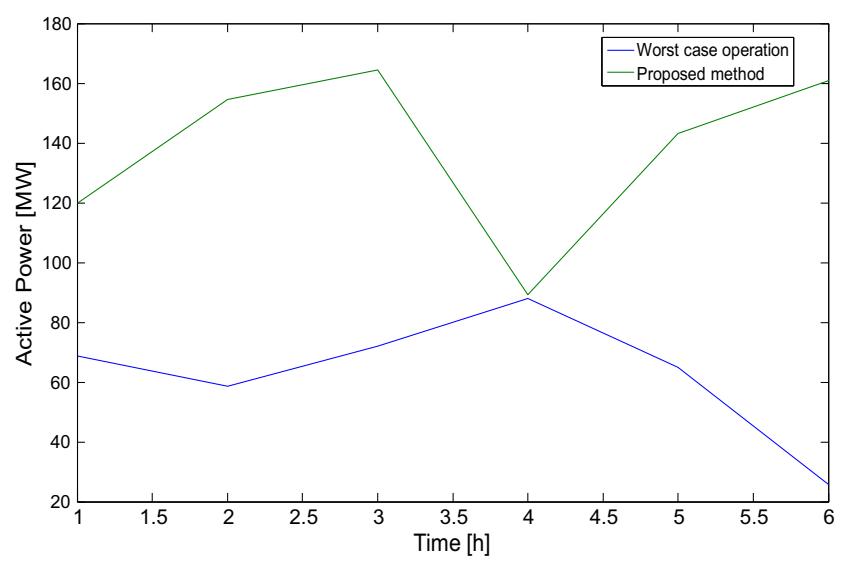

Fig. 9 Active power profile injected by the largest wind generator 


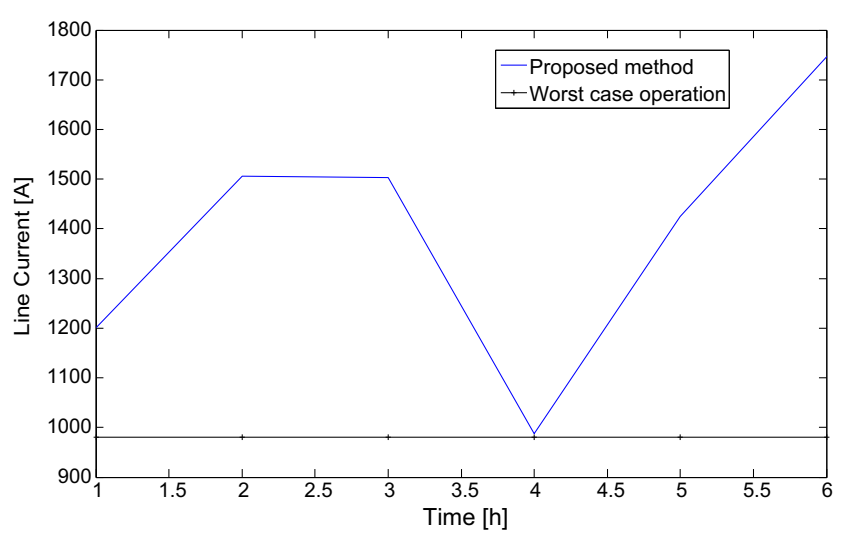

Fig. 10 Load current profile of the congested line

By analyzing these data it is worth observing that the solution computed by the CPPS framework sensibly improves the dispatch of the available wind energy. This has been obtained by reliably improving the loading of the congested line, which is extremely under-utilized in the traditional worst-case operation as confirmed by Fig. 11. By analyzing this figure it is important to note that the hotspot temperature profile computed by the calibrated thermal model by using the measured data, is extremely conservative for the worst-case operation, and more realistic, but still conservative, by the CPPS framework.

\section{Cybersecurity Issues}

A comprehensive cyber-security analysis of the CPPS framework aimed at assessing the potential vulnerabilities of the architecture modules to cyber-attacks, and designing proper reactive process for detecting and mitigating these threats, represent an essential requirement for an effective

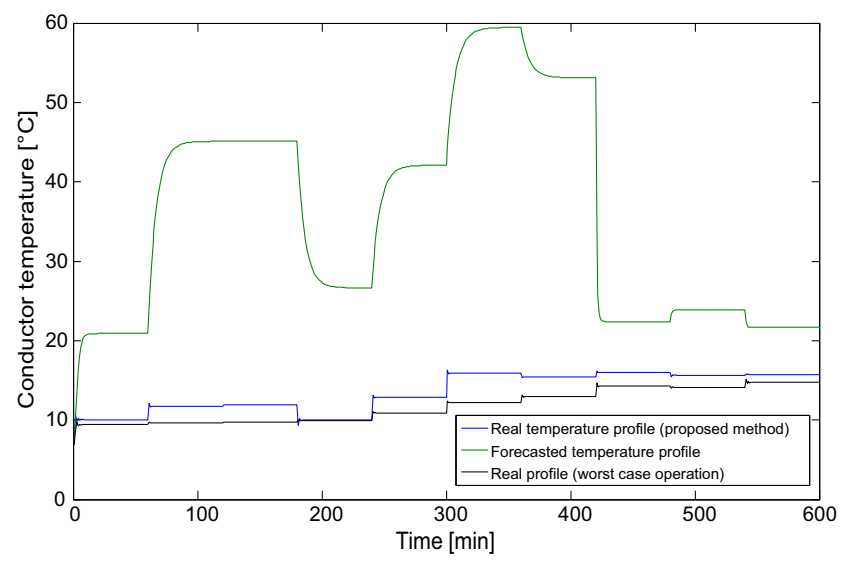

Fig. 11 Hot-spot temperature profile of the congested line deployment of the proposed architecture on real transmission networks. This issue is currently under investigation by the authors.

The first results obtained in this context confirmed that the wireless sensor network deployed along the line route to acquire the conductor temperature represents one of the most vulnerable module of the CPPS framework. In this context the effects of multiple cyber-attacks have been analyzed, e.g. the Wormhole attack [25], and in several attack scenario it has been observed an hindering of the information routing in the sensor network, which induced a collapse of the communication network.

\section{Concluding Remarks}

The paper proposed a decentralized and proactive CPPS architecture for integrated smart transmission control, modeling and monitoring. The proposed framework is based on an extendible set of interactive information services deployed according to the Web Services paradigm, which makes the CPPS platform-language- and vendorindependent. The core component of the proposed holistic framework is the Smart Grid Processing Engine, which is responsible for the execution of the CPPS functions in a geographically distributed scenario. It includes a network of remotely controlled units distributed in the most critical sections of the transmission network for data acquisition and advanced thermal protective functionalities; dedicated webservices aimed at interacting with power systems legacy application (e.g. WAMPAC, SCADA, EMS); a grid computing based solution engine for the on-line computations needed to implement the CPPS functions, and a web based interface for graphical synoptic, data storage and reporting.

These features contribute to improve the interoperability and the integration level of monolithic and hard to customize control and monitoring functions, as far as the flexibility in managing evolutionary changes in the structure and implementation of the internals of each CPPS function. Moreover, it allows developing content-based data extraction and aggregation from a host of pervasive sensors network and to exploit distributed embedded computing resources aimed at solving large-scale problems. These benefits have been confirmed by the first experimental results obtained on a real thermal-congested power system operating in the presence of a massive pervasion of renewable power generators.

\section{References}

1. European Commission (2014) A policy framework for climate and energy in the period from 2020 to 2030, white paper 
2. Madani V, King RL (2007) Strategies and applications to meet grid challenges and enhance power system performance. In: Proceedings of the IEEE power engineering society general meeting, pp 1-9

3. Giri J, Parashar M, Trehern J, Madani V (2012) The situation room: control center analytics for enhanced situational awareness. IEEE Power Energ Mag 10(5):24-39

4. Power System Engineering Research Center (2012) Cyber phisical systems for security of smart grids, white paper

5. Xinghuo Y, Cecati C, Dillon T, Simões MG (2011) The new frontier of smart grids. IEEE Ind Electron Mag 5(3):4963

6. de Mues MO, Alvarez A, Espinoza A, Garbajosa J (2011) Towards a distributed intelligent ICT architecture for the smart grid. In: 9th IEEE international conference on industrial informatics (INDIN 2011), pp 745-749

7. Georg H, Muller S, Rehtanz C, Wietfeld C (2014) Analyzing cyber-physical energy systems: The INSPIRE cosimulation of power and ICT systems using HLA. IEEE Trans Ind Inf 10(4):2364-2373

8. Hahn A, Ashok A, Sridhar S, Govindarasu M (2013) Cyberphysical security testbeds: architecture, application, and evaluation for smart grid. IEEE Trans Smart Grid 4(2):847-855

9. Cirio D, Lucarella D, Giannuzzi G, Tuosto F (2011) Wide area monitoring in the Italian power system: architecture, functions and experiences. International Transation on Electrical Energy Systems 21(4):1541-56

10. Giannuzzi G, Lauria D, Pisani C, Villacci D (2015) Real-time tracking of electromechanical oscillations in ENTSO-e Continental European Synchronous Area. Int J of Electr Power 64:11471158. doi:10.1016/j.ijepes.2014.09.016

11. Zhu J (2003) Web services provide the power to integrate. IEEE Power Energ Mag 1(6):40-49

12. Vaccaro A, Popov M, Villacci D, Terzija V (2011) An integrated framework for microgrids modeling, control, communication and verification. Invited Paper - IEEE Proceedings 99(1):119132

13. Morante Q, Ranaldo N, Vaccaro A, Zimeo E (2006) Pervasive grid for large-scale power systems contingency analysis. IEEE Trans Ind Inf 2(3):165-175
14. Giannuzzi G, Lauria D, Pisani C, Villacci D (2015) Real time tracking of electromechanical oscillations in ENSTO-e Continental European Synchronous Area. ELSEVIER Int J Electr Power Energy 64:1147-1158

15. Terzija V, Cai D, Valverde G, Regulski P, Vaccaro A, Osborne M, Fitch J Flexible wide area monitoring, protection and control applications in future power networks. In: The 10th institution of engineering and technology conference on developments in power system protection DPSP 201029 March - 1, April, 2010, The Hilton Deansgate, Manchester, UK

16. Vaccaro A, Villacci D (2008) An adaptive smart sensor network for overhead lines thermal rating prediction. Int $\mathrm{J}$ Emerg Electr Power Syst 9(4):2

17. Di Santo M, Ranaldo N, Villacci D, Zimeo E (2004) Performing security analysis of large scale power systems with a broker-based computational grid. In: Proceedings of ITCC, Las Vegas, USA

18. Di Santo M, Ranaldo N, Vaccaro A, Zimeo E (2004) Java-based distributed architectures for intensive computations in electrical grids

19. Vaccaro A, Mercogliano P, Schiano P, Villacci D (2011) An adaptive framework based on multi-model data fusion for one day ahead wind power forecasting. Electr Power Syst Res 81(3):775-782

20. European Centre for Medium-Range Weather Forecasts, IFS documentation CY33r1. On-line available http://www.ecmwf.int/ research/ifsdocs/CY33r1/index.html

21. Torelli F, Vaccaro A A novel state estimation paradigm based on the dynamic systems theory. In: Computational intelligence applications in smart grids, edited by Imperial College Press

22. Torelli F, Vaccaro A (2014) A generalized computing paradigm based on artificial dynamic models for mathematical programming. Soft Computing-Springer 18(8):1561-1573

23. Banakar H, Alguacil N, Galiana FD (2005) Electrothermal coordination part I: theory and implementation schemes. IEEE trans on Power Syst 20(2):798-805

24. Banakar H, Alguacil N, Galiana FD (2005) Electrothermal coordination part II: case studies. Energy Syst 20(4):1738-1745

25. Vaccaro A, Villacci D, Osborne M, Fitch J, Terzija V The role of cooperative sensor networks in wide area power systems communication. In: The 10th Institution of Engineering and Technology Conference on Developments in Power System Protection DPSP 201029 March - 1 April 2010, Manchester, UK 